\title{
-NOTES-
}

\section{NUMERICAL QUADRATURE OF SOME IMPROPER INTEGRALS*}

\author{
Br H. SERBIN (Purdue University)
}

1. Introduction. Problems in potential theory, such as occur in aerodynamics, may be formulated in terms of integrals which are usually singular. An explicit solution of such equations is rarely feasible and one is compelled to turn to numerical (approximate) methods. One such method, exemplified by Multhopp's solution [1] of the equation of the lifting line, depends on a quadrature formula of the singular integral. In the following analysis, we derive several formulae related to Multhopp's.

2. Parseval's identity. Let $\theta$ be a variable ranging on the interval $(-\pi, \pi)$, let $\theta_{r}, \phi_{r}=r \pi / N$ where $r=0, \pm 1, \cdots, \pm(N-1), N$ and $N$ is a positive integer, and consider the formal Fourier series on the interval $(-\pi, \pi)$

$$
f(\theta)=\sum a_{n} \cos n \theta+\sum b_{n} \sin n \theta, \quad g(\theta)=\sum a_{n}^{\prime} \cos n \theta+\sum b_{n}^{\prime} \sin n \theta .
$$

The $N$-th section of a Fourier series will be defined by writing

$$
f_{N}(\theta)=\sum_{0}^{N} a_{n} \cos n \theta+\sum_{1}^{N-1} b_{n} \sin n \theta .
$$

Introduce the notation

$$
[f(\theta), g(\theta)]=(2 \pi)^{-1} \int_{-\pi}^{\pi} f(\theta) g(\theta) d \theta, \quad[f(\theta), g(\theta)]_{N}=(2 N)^{-1} \sum_{r} f\left(\theta_{r}\right) g\left(\theta_{r}\right),
$$

where the summation is over all $r$. Parseval's relation is

$$
[f(\theta), g(\theta)]=a_{0} a_{0}^{\prime}+\frac{1}{2} \sum_{n=1}^{\infty}\left(a_{n} a_{n}^{\prime}+b_{n} b_{n}^{\prime}\right)
$$

and the corresponding relation for finite harmonic series is

$$
\left(f_{N}, g_{N}\right)_{N}=a_{0} a_{0}^{\prime}+\frac{1}{2} \sum_{1}^{N-1}\left(a_{n} a_{n}^{\prime}+b_{n} b_{n}^{\prime}\right)+a_{N} a_{N}^{\prime} .
$$

Comparing Eqs. (1) and (2), one obtains the

Theorem

$$
\left(f_{N}, g_{N}\right)=\left(f_{N}, g_{N}\right)_{N}-\frac{1}{2} a_{N} a_{N}^{\prime} .
$$

The theorem establishes a means of replacing an integral by a finite sum. When $f_{N}$ and $g_{N}$ are replaced by integrable functions, then the right side of (3) is an approximation to $(f, g)$.

For a fixed $N$, the functions $f_{N}(\theta)$ form a linear manifold in which two functions $f_{N}(\theta)$ and $g_{N}(\theta)$ are equal if and only if for every $r$

$$
f_{N}\left(\theta_{r}\right)=g_{N}\left(\theta_{r}\right) \text {. }
$$

${ }^{*}$ Received July 15, 1953. 
Equality will be used only in this sense below. We shall be concerned with linear transformations $T$ which transform this manifold into itself.

As an example, consider the identity transformation $T$ defined by

$$
T\left[f_{N}(\theta)\right]=f_{N}(\theta) .
$$

Since $T(1)=1, T(\cos n \theta)=\cos n \theta, T(\sin n \theta)=\sin n \theta$, one can verify, using the Fourier orthogonality relations, that $T$ may be represented by writing

$$
\begin{aligned}
T f_{N}(\theta) & =\left[1+2 \sum_{1}^{N}(\cos n \theta \cos n \phi+\sin n \theta \sin n \phi), f_{N}(\phi)\right], \\
{\left[T f_{N}(\theta)\right]_{\theta-\theta_{r}}=} & {\left[1+2 \sum_{1}^{N} \cos n \theta_{r} \cos n \phi+2 \sum_{1}^{N-1} \sin n \theta_{r} \sin n \phi, f_{N}(\phi)\right] . }
\end{aligned}
$$

Applying the theorem above,

$$
\left[T f_{N}(\theta)\right]_{\theta=\theta_{r}}=\left[1+2 \sum_{1}^{N} \cos n \theta_{r} \cos n \phi+2 \sum_{1}^{N-1} \sin n \theta_{r} \sin n \phi, f_{N}(\phi)\right]_{N}-(-1)^{r} a_{N} .
$$

3. An integral in the theory of airfoil sections. An integral which occurs repeatedly in the theory of airfoil sections defines a transformation

$$
T[f(\theta)]=(2 \pi)^{-1} \int_{-\pi}^{\pi} \cot \frac{1}{2}(\theta-\phi) f(\phi) d \phi
$$

where the integral is to be understood in the sense of "principal value":

$$
\int_{-\pi}^{\pi}=\operatorname{Lim}_{\epsilon \rightarrow 0}\left[\int_{-\pi}^{\theta-\epsilon}+\int_{\theta+\epsilon}^{\pi}\right] \text {. }
$$

If $f(\theta)$ is regarded as being defined on the unit circle then $T[f(\theta)]$ is that harmonic conjugate of $f(\theta)$ which has average value zero. One has

$$
T(\cos n \theta)=\sin n \theta, \quad T(\sin n \theta)=-\cos n \theta .
$$

Hence one has the following representation of $T$

$$
T\left[f_{N}(\theta)\right]=\left[2 \sum_{r_{1}}^{N}(\sin n \theta \cos n \phi-\cos n \theta \sin n \phi), f_{N}(\phi)\right] .
$$

But [ $\left.\sin N \phi, f_{N}(\phi)\right]=0$ and the summation of the second term under the bracket need extend only to $N-1$. Applying the theorem and noting that $\sin N \phi_{r}=0$, one obtains

Use the identity

$$
\begin{aligned}
T\left[f_{N}(\theta)\right] & =\left[2 \sum_{1}^{N}(\sin n \theta \cos n \phi-\cos n \theta \sin n \phi), f_{N}(\phi)\right]_{N} \\
& =\left[2 \sum_{1}^{N} \sin n(\theta-\phi), f_{N}(\phi)\right]_{N} .
\end{aligned}
$$

$$
\sum_{1}^{N} \sin n(\theta-\phi)=\cot \frac{1}{2}(\theta-\phi), 0
$$

according as $\theta-\phi$ is an odd or even multiple of $\pi / N$. Then

$$
T\left[f_{N}(\theta)\right]=\left[2 \cot \frac{1}{2}(\theta-\phi), f_{N}(\phi)\right]_{N}^{\prime},
$$


where the prime indicates that the summation on $\phi$ is taken over those values of $\phi$ which differ from $\theta$ by an odd multiple of $\pi / N$. Explicitly,

$$
T\left[f_{N}(\theta)\right]_{\theta=\theta_{r}}=N^{-1} \sum_{r+s \text { odd }}^{\prime} \cot \frac{1}{2}\left(\theta_{r}-\phi_{s}\right) f_{N}\left(\phi_{s}\right) .
$$

4. Example. To illustrate the preceding result, consider an infinitely thin stationary airfoil $y=y(x),-1 \leqq x \leqq 1$, immersed in a perfect fluid of uniform remote velocity $(1,0)$. Let $\alpha(x)$ represent the function $d y / d x$ and let $\gamma(x)$ be the vorticity per unit chord. Then one has the equation $[2, \mathrm{p} .88]$

$$
\alpha(x)=(2 \pi)^{-1} \cdot \int_{-1}^{+1}\left(x-x^{\prime}\right)^{-1} \gamma\left(x^{\prime}\right) d x^{\prime} .
$$

Make the transformations $x=\cos \theta, x^{\prime}=\cos \theta^{\prime}$ and multiply through by $\sin \theta$. Using the same functional symbols $\alpha$ and $\gamma$, one has

$$
\begin{aligned}
\alpha(\theta) \sin \theta & =-(2 \pi)^{-1} \int_{0}^{\pi} \sin \theta\left(\cos \theta-\cos \theta^{\prime}\right)^{-1}\left[\gamma\left(\theta^{\prime}\right) \sin \theta^{\prime}\right] d \theta^{\prime} \\
& =(4 \pi)^{-1} \int_{0}^{\pi}\left[\cot \frac{1}{2}\left(\theta-\theta^{\prime}\right)+\cot \frac{1}{2}\left(\theta+\theta^{\prime}\right)\right]\left[\gamma\left(\theta^{\prime}\right) \sin \theta^{\prime}\right] d \theta^{\prime} .
\end{aligned}
$$

Extend the range of definition of $\alpha(\theta)$ to the interval $(-\pi, 0)$ by defining $\alpha(\theta)$ as an even function and extend $\gamma(\theta)$ as an odd function. Then (7) becomes

$$
2 \alpha(\theta) \sin \theta=(2 \pi)^{-1} \int_{-\pi}^{\pi}\left[\cot \frac{1}{2}\left(\theta-\theta^{\prime}\right)\right]\left[\gamma\left(\theta^{\prime}\right) \sin \theta^{\prime}\right] d \theta^{\prime}=T(f(\theta)]
$$

where $f(\theta)=\gamma(\theta) \sin \theta$ and $T$ has the meaning of Eq. (5).

In airfoil theory, one invokes the Kutta condition ( $\gamma$ is finite at the trailing edge $x=1$, i.e., $\theta=0$ ) so that $f(0)=0$. Applying Eq. (6) and reversing the argument leading to $(7)$,

$$
\begin{gathered}
T[f(\theta)]_{\theta=\theta_{r}} \cong N^{-1} \sum^{\prime} \cot \frac{1}{2}\left(\theta_{r}-\phi_{s}\right) f\left(\phi_{s}\right) \\
-\frac{1}{2} T[f(\theta)]_{\theta-\theta_{r}}=N^{-1}\left\{\sum_{s=1}^{N-1} \frac{\sin \theta_{r}}{\cos \theta_{r}-\cos \phi_{s}} f\left(\phi_{s}\right)+\frac{1}{2} \epsilon_{r N} \tan \frac{1}{2} \theta_{r} f\left(\phi_{N}\right)\right\}
\end{gathered}
$$

where $\epsilon_{r N}=0$ or 1 according as $N-r$ is even or odd. Comparing (8) and (9) and simplifying, one obtains an approximate equation

$-\alpha\left(\theta_{r}\right) \cong N^{-1}\left\{\sum_{s=1}^{N-1}\left(\cos \theta_{r}-\cos \phi_{s}\right)^{-1} f\left(\phi_{s}\right)-\frac{1}{2} \epsilon_{r N}\left(1+\cos \theta_{r}\right)^{-1} f\left(\phi_{N}\right)\right\}, \quad \theta_{r} \neq 0, \pi$.

Returning to the original $x$-coordinate system

$$
\alpha\left(x_{r}\right) \cong-N^{-1}\left\{\sum_{s=1}^{N-1}\left(x_{r}-x_{s}\right)^{-1} f\left(x_{s}\right)-\frac{1}{2} \epsilon_{r N}\left(1+x_{r}\right)^{-1} f(-1)\right\}
$$

where $\left|\cos \theta_{r}\right| \neq 1$.

Equation (10) furnishes $N-1$ equations in the $N$ unknowns $f\left(x_{1}\right), \cdots, f\left(x_{N}\right)$. One additional equation follows upon the observation that the left side of Eq. (8) contains sine terms only up to order $N-1$. Hence $f(\theta)$ must contain cosine terms only to order $N-1$. Therefore

$$
a_{N}=(2 N)^{-1} \sum \cos N \theta_{r} f\left(\theta_{r}\right)=0,
$$


which leads to the equivalent equation

$$
-f\left(x_{1}\right)+f\left(x_{2}\right)-f\left(x_{3}\right)+\cdots \pm\left(\frac{1}{2}\right) f(-1)=0 .
$$

Equations (10 and (11) furnish a system of $N$ equations in $N$ unknowns.

As a numerical example, consider the straight line airfoil inclined at a small incidence $\alpha$ to the airstream. Then $\alpha(x)=-\alpha$ and Eqs. (10) and (11), for $N=3$, give the system

$$
f_{2}=3 \alpha, \quad-f_{1}+f_{3}=3 \alpha, \quad-f_{1}+f_{2}-\frac{1}{2} f_{3}=0,
$$

where $f_{r} \equiv f\left(x_{r}\right)$. Solution gives $f_{1}=\alpha, f_{2}=3 \alpha, f_{3}=4 \alpha$. Since $f_{0}=0$, one may interpolate harmonically with respect to $\theta$ using $N=3$, obtaining

$$
\begin{aligned}
& f(\theta)=2 \alpha(1-\cos \theta), \\
& \gamma(\theta)=2 \alpha(1-\cos \theta) / \sin \theta=2 \alpha(1-x)^{1 / 2}(1+x)^{-1 / 2},
\end{aligned}
$$

which is the exact result.

The preceding example is not intended as a substitute for the more direct method usually used in solving the problem of thin airfoil sections. However, it does illustrate the utility of the quadrature formula (6) in the evaluation of integrals (5) which appear in other connections (thick airfoil theory).

5. An integral in the theory of the lifting line. As the next case, consider the transformation

$$
T[f(\theta)]=(2 \pi)^{-1} \int_{-\pi}^{\pi} \cot \frac{1}{2}(\theta-\phi) f^{\prime}(\phi) d \phi,
$$

where the prime denotes differentiation. One has, as before,

$$
T\left[f_{N}(\theta)\right]=\left[2 \sum_{1}^{N} \sin n(\theta-\phi), f_{N}^{\prime}(\phi)\right] .
$$

Integration by parts gives

$$
T\left[f_{N}(\theta)\right]=\left[2 \sum_{1}^{N} n \cos n(\theta-\phi), f_{N}(\phi)\right]
$$

and application of the theorem

$$
T\left[f_{N}(\theta)\right]_{\theta=\theta_{r}}=\left[2 \sum_{1}^{N} n \cos n\left(\theta_{r}-\phi\right), f_{N}(\phi)\right]_{N}-N\left(\cos N \theta_{r}\right) a_{N} .
$$

Use the relation

$$
a_{N}=(2 N)^{-1} \sum_{s=0}^{2 N-1}(-1)^{s} f_{N}\left(\theta_{s}\right)
$$

and the identity

$$
2 \sum_{1}^{N} n \cos n x=N, \quad-N-\csc ^{2} x / 2, \quad N+N^{2}, \quad(x=k \pi / N),
$$

according as $k$ is even and different from zero, $k$ is odd, or $k=0$. Then (13) takes the form, after simplification,

$$
T\left[f_{N}(\theta)\right]_{\theta-\theta_{r}}=\frac{1}{2} N f_{N}\left(\theta_{r}\right)-(2 N)^{-1} \sum_{\zeta}^{\prime} \csc ^{2} \frac{1}{2}\left(\theta_{r}-\phi_{\triangleleft}\right) f_{N}\left(\phi_{\triangleleft}\right) .
$$


It is interesting to compare (14) with the result of integrating (12) formally by parts, disregarding the singularity. The integration leads to a divergent integral

$$
T[f(\theta)]=-(4 \pi)^{-1} \int_{-\pi}^{\pi} \csc ^{2} \frac{1}{2}(\theta-\phi) f(\phi) d \phi .
$$

The sum in Eq. (14) is a special kind of "Riemann sum" corresponding to the integral (15).

Equation (14) may be applied to derive Multhopp's quadrature formula. Consider a finite wing of unit semi-span immersed in a uniform (perfect) stream of unit velocity. Let $y$ and $y^{\prime}$ represent the spanwise coordinate; let $\Delta \alpha(y)$ and $\Gamma(y)$ represent the induced angle of attack and circulation, respectively, at station $y$.

The induced angle is given by [2, p. 135]

where

$$
\Delta \alpha(y)=(4 \pi)^{-1} \int_{-1}^{+1} \frac{d \Gamma / d y^{\prime}}{y-y^{\prime}} d y^{\prime}
$$

$$
\Gamma(-1)=\Gamma(1)=0 .
$$

Put $y=-\cos \phi, y^{\prime}=-\cos \phi^{\prime}$. Use the same functional notation and extend $\Delta \alpha(\phi)$ and $\Gamma(\phi)$ into the range $-\pi<\phi, \phi^{\prime}<0$ as even and odd functions respectively. In a way similar to that used in deriving Eq. (8), one obtains

$$
4 \Delta \alpha(\phi) \sin \phi=T[\Gamma(\phi)],
$$

where $T$ is used in the sense of Eq. (12). Using (17), one can write the sum in (14).

$$
\sum^{\prime} \csc ^{2} \frac{1}{2}\left(\theta_{r}-\phi_{s}\right) \Gamma\left(\phi_{s}\right)=\sum_{0<s<N}^{\prime}\left\{\csc ^{2} \frac{1}{2}\left(\theta_{r}-\phi_{s}\right) \Gamma\left(\phi_{s}\right)+\csc ^{2} \frac{1}{2}\left(\theta_{r}+\phi_{s}\right) \Gamma\left(-\phi_{s}\right)\right\} .
$$

Replace $\Gamma\left(-\phi_{s}\right)$ by $-\Gamma\left(\phi_{s}\right)$. Passing to trigonometric functions of double argument, one obtains

$$
\sum_{s}^{\prime} \csc ^{2} \frac{1}{2}\left(\theta_{r}-\phi_{s}\right) \Gamma\left(\phi_{s}\right)=4 \sum_{0<s<N}^{\prime} \sin \theta_{r} \sin \phi_{s}\left(\cos \theta_{r}-\cos \phi_{s}\right)^{-2} \Gamma\left(\phi_{s}\right) .
$$

Substituting this result in (14) and the result so obtained in (18), one obtains, after simplification,

$$
\Delta \alpha\left(\theta_{r}\right)=N \Gamma\left(\theta_{r}\right) /\left(8 \sin \theta_{r}\right)-(2 N)^{-1} \sum_{0<s<N}^{\prime} \sin \phi_{s}\left(\cos \theta_{r}-\cos \phi_{s}\right)^{-2} \Gamma\left(\phi_{s}\right) .
$$

Equation (19) is equivalent to Multhopp's quadrature formula. For numerical applications, the reader is referred to [1].

6. An integral over an infinite range. Define a transformation $T$ by

$$
T[f(x)]=\pi^{-1} \int_{-\infty}^{\infty}(x-y)^{-1} f(y) d y .
$$

Consider the related transformation

$$
T_{1}[f(x)]=\pi^{-1} \int_{-L}^{+L}(x-y)^{-1} f(y) d y,
$$

where $L$ is a positive quantity.

Make the transformation

$$
\pi x=L \theta, \quad \pi y=L \phi
$$


and write

$$
T_{1}(f(x))=F(\theta), \quad f(y)=f_{1}(\phi) .
$$

Then

$$
F(\theta)=\pi^{-1} \int_{-\pi}^{\pi}(\theta-\phi)^{-1} f_{1}(\phi) d \phi
$$

Write

$$
\theta-\phi=\frac{1}{2} \cot \frac{1}{2}(\theta-\phi)+L(\theta, \phi),
$$

where $L(\theta, \phi)$ is continuous in $(\theta, \phi)$. Then, using (6),

$$
\begin{gathered}
F(\theta)=(2 \pi)^{-1} \int_{-\pi}^{\pi} \cot \frac{1}{2}(\theta-\phi) f_{1}(\phi) d \phi+\pi^{-1} \int_{-\pi}^{\pi} L(\theta, \phi) f_{1}(\phi) d \phi, \\
F\left(\theta_{r}\right) \cong N^{-1} \sum_{s}^{\prime} \cot \frac{1}{2}\left(\theta_{r}-\phi_{s}\right) f_{1}\left(\phi_{s}\right)+\pi^{-1}(2 \pi / N) \sum_{s}^{\prime} L\left(\theta_{r}, \phi_{s}\right) f_{1}\left(\phi_{s}\right),
\end{gathered}
$$

where the second integral has been approximated by a Riemann sum. Combining terms and noting (24),

$$
\begin{aligned}
F\left(\theta_{r}\right) & =2 / N \sum_{s}^{\prime}\left(\theta_{r}-\phi_{s}\right)^{-1} f_{1}\left(\phi_{s}\right) \\
& =(2 / \pi) \sum_{-N \leq s<N}^{\prime}(r-s)^{-1} f\left(x_{s}\right), \quad x_{s}=s L / N .
\end{aligned}
$$

Equation (25) relates to (21). We conjecture that the modified quadrature formula

$$
T[f(x)]_{x=x_{r}} \cong(2 / \pi) \sum_{-\infty<s<\infty}^{\prime}(r-s)^{-1} f\left(x_{\diamond}\right),
$$

where $x_{s}=s \Delta x$, would be more suitable for Eq. (20). In fact, if one puts

$$
f(x)=\exp (i k x),
$$

then one can write the summation in Eq. (26) in the form

$$
-\exp \left(i k x_{r}\right) \sum_{\sigma \text { odd }>0} 2 i \sigma^{-1} \sin (\sigma k \Delta x)=-i \exp \left(i k x_{r}\right) g\left\{\log \frac{1+\exp (-i k \Delta x)}{1-\exp (-i k \Delta x)}\right\} .
$$

Here the symbol $g$ represents "imaginary part of" and the log function is suitably defined as a single-valued function. When $k \Delta x$ is not a multiple of $\pi$, then simplification of (27) and substitution into (26) gives the result

$$
T[f(x)]_{x=x_{r}}=i \exp \left(i k x_{r}\right),
$$

which is the exact result.

More generally, consider functions of the form

$$
f(x)=\int_{-K}^{K} g(k) \exp (i k x) d k
$$

where $g(k)$ is a continuous function and $K$ is a positive quantity less than $\pi / \Delta x$. The infinite series

$$
\sum^{\prime}(r-s) \exp \left(i k x_{r}\right), \quad x_{r}=r \Delta x
$$


summed over odd $s$, is uniformly convergent in $k$ because of Abel's lemma.

Hence

$(2 / \pi) \sum^{\prime}(r-s)^{-1} \int_{-K}^{K} g(k) \exp \left(i k x_{s}\right) d k=(2 / \pi) \int_{-K}^{K} g(k)\left[\sum^{\prime}(r-s)^{-1} \exp \left(i k x_{s}\right)\right] d k$

$$
=i \int_{-K}^{K} g(k) \exp \left(i k x_{r}\right) d k
$$

in view of (28). However, the transform of $f(x)$, Eq. (29) is given by

$$
T[f(x)]=\pi^{-1} \int_{-\infty}^{\infty}(x-y)^{-1} \int_{-K}^{K} g(k) \exp (i k y) d k d y .
$$

When $x$ is restricted to any finite interval, the order of integration may be interchanged provided that one interprets the singular integral with respect to $y$ as a "principal value". Hence

$$
\begin{aligned}
T[f(x)] & =\pi^{-1} \int_{-K}^{K} g(k) \int_{-\infty}^{\infty}(x-y)^{-1} \exp (i k y) d y d k \\
& =i \int_{-K}^{K} g(k) \exp (i k x) d k
\end{aligned}
$$

which agrees with (30). Therefore the quadrature formula (26) is exact for functions of the form (29) provided that

$$
K \Delta x<\pi
$$

\section{REFERENCES}

1. H. Multhopp, Die Berechnung der Auftriebsverteilung von Tragfiügeln, Lufo 15, 153-169 (1938); also available as British RTP Translation 2392.

2. H. Glauert, The elements of aerofoil and airscrew theory, Cambridge University Press, 1930.

\section{NOTE ON THE EXISTENCE AND DETERMINATION OF A VECTOR POTENTIAL*}

\section{By A. F. STEVENSON (Wayne University)}

The problem of finding a solution of the equation

$$
\operatorname{curl} \mathbf{F}=\mathbf{f} \text {, }
$$

where $\mathbf{f}$ is a given vector function of position and $\mathbf{F}$ is to be found, is a classical one, and one would hardly think that there was anything fresh to be said about the matter to-day. In connection with some recent work, however, the writer had occasion to look into the matter somewhat closely, and as a result of this it appeared that the usual treatments $^{1}$ are not satisfactory if $\mathrm{f}$ is only defined in a restricted region. Usually, in

*Received June 23, 1953.

'The following textbooks were consulted: R. Courant, Differential and integral calculus, vol. II (Nordeman, New York, 1944); E. Goursat, Mathematical analysis, vol. I (Ginn, New York, 1904); E. Picard, Traite d'analyse, vol. I (Gauthier-Villars, Paris, 1922); C. E. Weatherburn, Advanced vector analysis (Bell, London, 1928); H. Lass, Vector and tensor analysis (McGraw-Hill, New York, 1950). 